\title{
Avaliação da sarcopenia em idosos em extrema longevidade utilizando diferentes métodos e sua relação com o desempenho cognitivo
}

\section{Evaluation of sarcopenia in older adults in extreme longevity using different methods and its relationship to cognitive performance}

\author{
Dathália de Oliveira Andrade ${ }^{1}$, (D) Gustavo Henrique Martins Rodrigues Montalvão ${ }^{1}$, (D) Isadora Cecília Salgado Gama ${ }^{1}$, \\ (D) Mariana de Paula Santana ${ }^{1}$, (D) Bruna Moretti Luchesi ${ }^{1}$, (D) Marcelo Kwiatkoski ${ }^{1}$
}

${ }^{1}$ Universidade Federal de Mato Grosso do Sul UFMS

\section{Correspondência}

Bruna Moretti Luchesi

E-mail: bruna.luchesi@ufms.br

Submetido: 05 Abril 2020

Aceito: 21 Outubro 2020

\section{Como citar}

Andrade NO, Montalvão GHMR, Gama ICS, Santana MP, Luchesi BM, Kwiatkoski M Avaliação da sarcopenia em idosos em extrema longevidade utilizando diferentes métodos e sua relação com o desempenho cognitivo. Acta Fisiatr. 2020;27(3):139-145

DOI: 10.11606/issn.2317-0190.v27i3a174634

\section{cc) $\$$}

\section{RESUMO}

A sarcopenia é altamente prevalente nos idosos, gerando desafios individuais e sociais para a saúde pública, o que torna o rastreio da mesma fundamental. Objetivo: Avaliar a relação entre massa muscular esquelética (MME) e circunferência da panturrilha (CP), índice de massa corporal (IMC) e desempenho cognitivo de idosos em extrema longevidade. Método: Pesquisa transversal e quantitativa com $n=69$ idosos com idade igual ou maior que 90 anos, atendidos no Sistema Único de Saúde. Coletaram-se dados sociodemográficos e antropométricos. O índice de massa muscular (IMM) foi calculado com base na MME, a qual foi estimada pela fórmula de Lee e colaboradores. A definição de sarcopenia utilizada foi a proposta por Janssen e colaboradores. Foi feita a medida da $\mathrm{CP}$, calculado o IMC, e o desempenho cognitivo foi avaliado pelo teste de Fluência Verbal (FV). A relação entre as variáveis foi analisada pelo teste de correlação de Spearman. Resultados: Todos os idosos foram considerados com sarcopenia severa de acordo com o IMM. Pela CP, 39,4\% dos homens e 52,8\% das mulheres apresentaram valores indicativos de sarcopenia; e pelo IMC, 33,3\% dos homens e $36,1 \%$ das mulheres possuem baixo peso corporal. As únicas variáveis que apresentaram relação significativa e direta foram IMC e CP, para homens e mulheres. Conclusão: Não foi identificada correlação entre MME e CP, IMC e desempenho cognitivo. Sugere-se que a avaliação da sarcopenia em idosos longevos seja realizada utilizando métodos mais fidedignos. A elevada prevalência da sarcopenia realça a urgência de um olhar direcionado ao grupo de idosos longevos.

Palavras-chave: Sarcopenia, Antropometria, Índice de Massa Corporal, Cognição, Idoso de 80 Anos ou mais

\begin{abstract}
Sarcopenia is highly prevalent in older adults, and generates several individual and social challenges for public health, which makes screening it fundamental. Objective: To evaluate the relationship between skeletal muscle mass (SMM) and calf circumference (CC), body mass index (BMI) and cognitive performance of older adults in extreme longevity. Method: A crosssectional and quantitative research was carried out with $n=69$ elderly people aged 90 years or older, treated at the Unified Health System. Sociodemographic and anthropometric data were collected. The muscle mass index (MMI) was calculated based on the SMM, which was estimated by the formula of Lee and collaborators. The definition of sarcopenia used was proposed by Janssen and collaborators. CC was measured, BMI was calculated, and cognitive performance was assessed by the Verbal Fluency (VF) test. The relationship between the variables was analyzed using Spearman's correlation test. Results: All older adults evaluated were considered to have severe sarcopenia according to the MMI. According to the CC, $39.4 \%$ of men and $52.8 \%$ of women had indicative values for sarcopenia; and by BMI, $33.3 \%$ of men and $36.1 \%$ of women have low body weight. The only variables that showed a significant and direct relationship were $\mathrm{BMI}$ and $\mathrm{CC}$, for men and women. Conclusion: No correlation was found between SMM and CC, BMI and cognitive performance. The evaluation of sarcopenia in long-lived elderly should be carried out using methods that are more reliable. The high prevalence of sarcopenia highlights the urgency of looking at the group of extreme longevity.
\end{abstract}

Keywords: Sarcopenia, Anthropometry, Body Mass Index, Cognition, Aged, 80 and over 


\section{INTRODUÇÃO}

O processo de envelhecimento implica mudanças individuais, cumulativas e inevitáveis associadas à idade; modificações essas relacionadas à influência genética, mas principalmente a fatores ambientais e questões sociais. ${ }^{1} \mathrm{Em}$ 2017, o Brasil contava com 1,81\% da população octogenária, nonagenária e centenária, sendo que a projeção para 2030 é que essa população suba para $2,93 \%$ do total. ${ }^{2}$ Nesse contexto, ao mesmo tempo em que o aumento da longevidade humana representa uma conquista, também traz uma responsabilidade para os gestores públicos e para sociedade, demandando investimentos que promovam a autonomia e o bem-estar dos idosos. $^{3}$

Após alcançar a idade de 40 anos, adultos saudáveis perdem aproximadamente $8 \%$ de sua massa muscular a cada 10 anos, ocorrendo um declínio de em média 24\% dos músculos até os 70 anos, o que acelera para $15 \%$ por década após essa idade. ${ }^{4}$ Nesse sentido, encontra-se o conceito de sarcopenia que, de acordo com o European Working Group on Sarcopenia in Older People (EWGSOP), é definido como a síndrome caracterizada pela perda progressiva e generalizada de massa muscular esquelética (MME) e força, com consequências adversas, tais como deficiência física, qualidade baixa de vida e até morte. ${ }^{5}$

Como fatores de risco dessa condição, ressaltam-se circunstâncias que diminuem a atividade muscular, a exemplo de sedentarismo e hospitalização, fatores nutricionais que influenciam no suporte energético e na saúde dos músculos, além de condições inflamatórias crônicas e transtornos metabólicos, como distúrbios endócrinos e falência avançada de órgãos vitais. ${ }^{4}$

Estudos evidenciam que o índice de massa muscular (IMM), calculado a partir da MME pode predizer a sobrevida em adultos mais velhos. ${ }^{6,7}$ A sarcopenia é relacionada com diversas decorrências negativas graves para a saúde, principalmente incapacidade, fragilidade e quedas. ${ }^{8}$ Uma análise realizada durante dois anos com pessoas de 80 anos ou mais de idade revelou um índice três vezes maior de quedas em idosos sarcopênicos em relação aos sem essa condição. ${ }^{9}$

Em um estudo observacional realizado com idosos de 70 anos de idade ou mais na Itália, destacou-se que a sarcopenia era altamente prevalente entre os residentes de instituições, e associada a um risco consideravelmente aumentado de mortalidade por todas as causas. ${ }^{10}$

Além de ocasionar esses desfechos negativos, o estado sarcopênico também pode estar ligado a um maior risco de comprometimento cognitivo, independentemente da população em estudo, da definição de sarcopenia e do dano à cognição. ${ }^{11,12}$ No entanto, vislumbram-se inúmeras lacunas na literatura sobre essa associação, como o mecanismo biológico subjacente, ${ }^{12}$ somado a quais componentes da sarcopenia (massa muscular, força, condicionamento físico) se relacionam com domínios cognitivos. ${ }^{13}$ Desse modo, o presente estudo apresenta substancial relevância científica, não só por enriquecer a literatura nacional sobre a temática, mas também ao considerar a extrema longevidade nessa discussão.

Dentre as técnicas de avaliação de $M M E$, destacam-se a absorciometria radiológica de dupla energia (DXA), a tomografia computadorizada e a ressonância magnética. ${ }^{14}$
Relativamente a essas modalidades de imagem, a DXA consiste no método mais usado para estimar a perda de MME, devido às vantagens de precisão, rapidez no exame e baixa dose de radiação. ${ }^{15}$

Contudo, devido à falta de portabilidade e elevados custos dos equipamentos, essa tecnologia pode se revelar imprópria para estudos epidemiológicos e o cuidado na atenção básica. Nesse contexto, a equação preditiva de Lee e colaboradores atua como ferramenta fundamental para o diagnóstico de sarcopenia. Um estudo desenvolvido por Rech et al. ${ }^{16}$ identificou alta correlação entre a massa muscular estimada pela fórmula e a obtida pela DXA em idosos brasileiros.

Em relação ao baixo custo e facilidade de aplicação, a medição da circunferência da panturrilha (CP) também merece evidência na detecção da sarcopenia, já que existe uma correlação positiva da CP com a MME. ${ }^{17,18}$ Outro parâmetro antropométrico bastante difundido é o índice de massa corporal (IMC), o qual está associado à sarcopenia quando é baixo. ${ }^{19}$

Devido à transição demográfica, a sarcopenia sugere desafios à saúde pública, tanto na esfera individual, devido aos desfechos adversos; quanto na esfera social, pelo ônus atribuído à hospitalização e ao cuidado geriátrico. ${ }^{20}$ Nesse sentido, torna-se imprescindível a busca por ferramentas eficazes e acessíveis de detecção precoce da sarcopenia, a fim de prevenir seus efeitos negativos.

\section{OBJETIVO}

O objetivo da pesquisa consiste em avaliar a relação entre Massa Muscular Esquelética (MME) e Circunferência da Panturrilha (CP), Índice de Massa Corporal (IMC) e desempenho cognitivo de idosos em extrema longevidade.

\section{MÉTODO}

A pesquisa possui desenho transversal e foi baseada no método quantitativo de investigação. Foi realizada no município de Três Lagoas-MS em que, de acordo com o censo de 2010, havia uma população idosa ( $\geq 60$ anos) correspondente a $9,8 \%$ do total da cidade. ${ }^{21}$

Compuseram a população do estudo, idosos em extrema longevidade (90 anos ou mais) atendidos no Sistema Único de Saúde (SUS) da cidade no ano anterior ao início da coleta de dados (de novembro de 2018 a novembro de 2019). Para a amostra, os critérios de inclusão foram: ter idade maior ou igual a 90 anos, ter sido atendido por algum ponto da Rede de Atenção à Saúde do SUS do município no ano anterior, ser capaz de responder às questões da entrevista, e morar na zona urbana do município. Foram excluídos os idosos que não foram encontrados em suas casas após duas visitas.

Após busca no sistema de cadastro dos usuários do SUS da cidade, foram listados 345 idosos atendidos no ano anterior. Não foi possível visitar 183 idosos, sendo 10 por erro no cadastro, 57 por óbito, 14 por residirem na zona rural e 102 por mudança de endereço, endereço não encontrado ou inexistente.

Os 162 idosos restantes foram visitados, sendo que 62 se recusaram a participar da pesquisa, quatro eram incapazes de responder ao questionário e sete estavam em isolamento social, não sendo possível entrevistá-los. 
Foram avaliados 89 idosos, porém, não foi possível aferir peso e estatura de 20 idosos, os quais foram excluídos das análises do presente estudo por não completarem as variáveis de interesse. A amostra final foi de $n=69$ idosos em extrema longevidade.

A coleta de dados foi domiciliar, conduzida por avaliadores capacitados e aconteceu no período de dezembro de 2019 a fevereiro de 2020. Foram coletados dados sociodemográficos (sexo, idade, escolaridade, estado civil, e renda) e as variáveis de interesse avaliadas por meio dos instrumentos descritos abaixo. Ressalta-se que todas as medidas antropométricas foram coletadas em triplicata, e utilizado o resultado da média aritmética das mesmas.

A MME foi calculada com base na fórmula, de Lee e colaboradores, validada para idosos brasileiros, ${ }^{14,16}$ que leva em consideração a estatura do idoso, a massa corporal, o sexo, a idade e a etnia (asiático, afrodescendente ou caucasiano):

MME (quilogramas $-\mathrm{Kg})=$ Estatura em metros* $\left(0,244^{*}\right.$

Massa Corporal) $+\left(7,8^{*}\right.$ Estatura em centímetros $)+\left(6,6^{*}\right.$ sexo $)-(0,098 *$ idade $)+($ etnia $-3,3)$

Onde: para sexo considera-se $1=$ homens e $0=$ mulheres, e para etnia 1,2= asiáticos, 1,4= afrodescendente e $0=$ caucasianos. A MME foi usada como base para calcular o índice de massa muscular (IMM), por meio da fórmula:

\section{$\operatorname{IMM}\left(\mathrm{Kg} / \mathrm{m}^{2}\right)=M M E(\mathrm{~kg}) /$ Estatura em metros, ao quadrado}

Para determinar se o idoso era ou não sarcopênico, foram utilizados como referência os valores propostos por Janssen et al. ${ }^{22}$ sendo:

- Mulheres: $\leq 5,75 \mathrm{~kg} / \mathrm{m} 2=$ sarcopenia severa; 5,76 - 6,75 $\mathrm{kg} / \mathrm{m} 2=$ sarcopenia moderada e; $\geq 6,76 \mathrm{~kg} / \mathrm{m} 2=$ massa muscular normal.

- Homens: $\leq 8,50 \mathrm{~kg} / \mathrm{m} 2=$ sarcopenia severa; 8,51 - 10,75 $\mathrm{kg} / \mathrm{m} 2=$ sarcopenia moderada $\mathrm{e} ; \geq 10,76 \mathrm{~kg} / \mathrm{m} 2=$ massa muscular normal.

A CP contemplou a medida na maior circunferência da perna, onde valores $<31 \mathrm{~cm}$ são indicativos de sarcopenia. ${ }^{23} \mathrm{O}$ IMC corresponde ao cálculo do peso $(\mathrm{kg}) /$ altura $(\mathrm{m})^{2}$. Foram considerados obesos os idosos com IMC $\geq 27 \mathrm{~kg} / \mathrm{m}^{2}$ e com baixo peso, os com IMC $\leq 22 \mathrm{~kg} / \mathrm{m}^{2}{ }^{23}$

Por fim, o desempenho cognitivo foi avaliado pelo teste de Fluência Verbal (FV) categorias animais, que consiste em solicitar que o idoso diga o máximo de animais dos quais se lembrar no tempo de um minuto. As notas de corte são: nove, para zero a sete anos de escolaridade; e 13 para alta escolaridade (oito anos ou mais). ${ }^{24}$

Os dados foram introduzidos em dupla entrada e validados em uma planilha do Microsoft Office Excel ${ }^{\mathrm{TM}}$. Foram exportados ao Statistical Package for the Social Science (SPSS) versão 25.0 para realização das análises.

Conduziu-se a análise descritiva das variáveis quantitativas com medidas de tendência central e variabilidade e das variáveis categóricas com tabelas de frequência, divididas entre homens e mulheres. Para comparar os dois grupos, utilizamos o teste de correlação de Spearman (duas variáveis quantitativas) ou o teste $U$ de Mann-Whitney (uma variável quantitativa e uma categórica).
Como todos os idosos foram classificados como sarcopênicos, a relação entre o IMM e as variáveis $\mathrm{CP}$, IMC e FV foi realizada com os dados na forma contínua, por meio do teste de correlação de Spearman. Valores de $p \leq 0,05$ foram considerados significativos. O projeto foi aprovado pelo Comitê de Ética em Pesquisa com Seres Humanos da Universidade Federal de Mato Grosso do Sul, CAAE: 21397119.0.0000.0021 e o Termo de Consentimento Livre e Esclarecido foi lido e assinado em duas vias pelos participantes antes do início da coleta.

\section{RESULTADOS}

A Tabela 1 evidencia os dados de caracterização, sarcopenia e fluência verbal dos idosos em extrema longevidade. Todos os idosos foram considerados com sarcopenia severa de acordo com o IMM. Pela CP, 39,4\% dos homens e $52,8 \%$ das mulheres apresentaram valores indicativos de sarcopenia. De acordo com o cálculo do IMC, 33,3\% dos homens e 36,1\% das mulheres possuem baixo peso corporal.

A amostra teve como características idade e IMM significativamente maiores para as mulheres em comparação aos homens. Quanto à FV a pontuação foi maior para os homens. Com relação ao estado civil, todas as mulheres avaliadas viviam sem companheiros, ao passo que $48,5 \%$ dos homens tinham companheiras.

Tabela 1. Caracterização de idosos em extrema longevidade quanto aos dados sociodemográficos, de sarcopenia e fluência verbal

\begin{tabular}{|c|c|c|c|c|c|c|c|}
\hline & \multicolumn{3}{|c|}{ Homens $(n=33)$} & \multicolumn{3}{|c|}{ Mulheres $(n=36)$} & \multirow[b]{2}{*}{ p-valor } \\
\hline & $\mathbf{N}$ & $\%$ & $\begin{array}{c}\text { Média } \\
\text { (dp) }\end{array}$ & $\mathbf{N}$ & $\%$ & $\begin{array}{c}\text { Média } \\
\text { (dp) }\end{array}$ & \\
\hline Idade & & & $92,5(4,3)$ & & & $94,4(3,2)$ & $0,011^{*}$ \\
\hline Escolaridade & & & $1,3(2,0)$ & & & $1,4(1,6)$ & 0,612 \\
\hline Estado civil & & & & & & & $0,000^{*}$ \\
\hline Com companheiro & 16 & 48,5 & & & & & \\
\hline Sem companheiro & 17 & 51,5 & & 36 & 100 & & \\
\hline Renda familiar (R\$) & & & $\begin{array}{l}2176,6 \\
(501,8)\end{array}$ & & & $\begin{array}{c}2989,0 \\
(2026,4)\end{array}$ & 0,233 \\
\hline IMM (Kg/m2) & & & $4,9(0,2)$ & & & $5,3(0,2)$ & $0,000^{*}$ \\
\hline Normal & - & & & & & - & \\
\hline Sarcopenia moderada & - & & & & & - & \\
\hline Sarcopenia severa & 33 & 100 & & & 36 & 100 & 1 \\
\hline $\mathrm{CP}(\mathrm{cm})$ & & & $31,6(3,0)$ & & & $30,9(4,8)$ & 0,428 \\
\hline Normal & 20 & 60,6 & & 17 & 47 & & 0,265 \\
\hline Alterada & 13 & 39,4 & & 19 & 53 & & \\
\hline $\mathrm{IMC}(\mathrm{Kg} / \mathrm{m} 2)$ & & & $24,4(4,3)$ & & & $23,5(5,2)$ & 0,49 \\
\hline Baixo peso & 11 & 33,3 & & 13 & 36 & & 0,882 \\
\hline Peso adequado & 11 & 33,3 & & 13 & 36 & & \\
\hline Obesidade & 11 & 33,3 & & 10 & 28 & & \\
\hline FV & & & $10,6(4,4)$ & & & $7,6(4,2)$ & $0,006^{*}$ \\
\hline Normal & 16 & 48,5 & & 8 & 22 & & $0,027^{*}$ \\
\hline Alterada & 17 & 51,5 & & 28 & 78 & & \\
\hline
\end{tabular}

$D p=$ desvio padrão; MME= Massa Muscular Esquelética; IMM= Índice de Massa Muscular $C P=$ Circunferência da Panturrilha; $I M C=$ Índice de Massa Corporal; FV = Fluência verbal 
As Tabelas 2 e 3 evidenciam os dados de correlação entre as variáveis, para os homens e para as mulheres, respectivamente. Em ambos os grupos, as únicas variáveis que apresentaram correlação significativa foram IMC e CP $(\rho=$ 0,$700 ; p=0,000$ para homens $\rho=0,815 ; p=0,000$ para mulheres). Não foi identificada relação entre IMM e CP, IMC e desempenho cognitivo para homens e para mulheres.

Tabela 2. Matriz de correlação de IMM, CP, IMC e FV de idosos em extrema longevidade do sexo masculino $(n=33)$

\begin{tabular}{|c|c|c|c|c|}
\hline Variáveis & IMM & $\mathrm{CP}$ & IMC & FV \\
\hline IMM & - & $\begin{array}{c}\rho=-0,017 \\
p=0,924\end{array}$ & $\begin{array}{l}\rho=0,231 \\
p=0,196\end{array}$ & $\begin{array}{c}\rho=-0,274 \\
p=0,123\end{array}$ \\
\hline CP & - & - & $\begin{array}{l}\rho=0,700 \\
p=0,000^{*}\end{array}$ & $\begin{array}{c}\rho=-0,040 ; \\
p=0,823\end{array}$ \\
\hline IMC & - & - & - & $\begin{array}{c}\rho=-0,236 \\
p=0,187\end{array}$ \\
\hline
\end{tabular}

$\rho=$ Rô Spearman; $I M M=$ Índice de Massa Muscular; $C P=$ Circunferência da Panturrilha; IMC= Índice de Massa Corporal; FV= Fluência verbal

Tabela 3. Matriz de correlação de IMM, CP, IMC e FV de idosos em extrema longevidade do sexo feminino $(n=36)$

\begin{tabular}{|c|c|c|c|c|}
\hline Variáveis & IMM & CP & IMC & $\mathrm{FV}$ \\
\hline IMM & - & $\begin{array}{c}\rho=-0,245 \\
\mathrm{p}=0,156\end{array}$ & $\begin{array}{l}\rho=0,012 ; \\
\mathrm{p}=0,944\end{array}$ & $\begin{array}{c}\rho=-0,172 \\
\mathrm{p}=0,332\end{array}$ \\
\hline CP & - & - & $\begin{array}{l}\rho=0,815 \\
p=0,000^{*}\end{array}$ & $\begin{array}{l}\rho=0,231 ; \\
p=0,182\end{array}$ \\
\hline IMC & - & - & - & $\begin{array}{c}\rho=-0,021 ; \\
\mathrm{p}=0,903\end{array}$ \\
\hline
\end{tabular}

$\rho=$ Rô Spearman; $I M M=$ Índice de Massa Muscular; $C P=$ Circunferência da Panturrilha; IMC= Índice de Massa Corporal; FV= Fluência verbal

\section{DISCUSSÃO}

De acordo com o IMM, todos os idosos foram considerados com sarcopenia severa. Pesquisas anteriores, ao utilizar esse mesmo critério, indicaram prevalências diversificadas, variando de 5 a $13 \%$ entre os septuagenários e de 11 a $50 \%$ entre os octogenários. ${ }^{25,26}$

Mesmo considerando como amostra o público acima de 60 anos, esses estudos não revelaram um percentual tão alto ou até mesmo próximo do encontrado no presente estudo.

A alta prevalência de sarcopenia em idosos longevos mostra quão relacionada está a idade com esse fator, dado já relatado anteriormente. ${ }^{27,28}$

Pela CP, a prevalência de sarcopenia se apresentou inferior ao IMM, sendo de $39,4 \%$ nos homens e $52,8 \%$ nas mulheres, e mostrou relação direta com o IMC em ambos os grupos. A CP tem sido relatada como uma medida confiável para a identificação precoce de sarcopenia, correlacionando-se fortemente com a massa muscular. ${ }^{18}$

Porém, mesmo que os estudos apontem uma elevada especificidade desse parâmetro, constatou-se sua baixa sensibilidade. ${ }^{29}$ Assim, o teste se configura como adequado para evitar diagnósticos incorretos e intervenções desnecessárias, porém, não é tão apurado para rastreio, o que explicaria a discrepância entre os resultados das avaliações.

Nesse sentido, destaca-se a importância de avaliações mais precisas, em especial para idosos longevos, desse quadro clínico tão presente com o avanço da idade. ${ }^{29}$ Os resultados em análise mostram que a identificação de sarcopenia a partir da CP pode ser falha.

O aumento da infiltração de tecido adiposo, entre as fibras musculares, intrinsecamente ligada ao envelhecimento ${ }^{30}$ pode ser uma justificativa plausível para a perda de confiabilidade dessa medida. Cabe, portanto, cautela ao se usar esse parâmetro na deteç̧ão de sarcopenia em idosos com idade extrema, uma vez que podem existir falsos-negativos.

$\mathrm{O}$ baixo peso evidenciado pelo IMC apresentou prevalência de $33,3 \%$ nos homens e $36,1 \%$ nas mulheres. Em estudos anteriores, o estado nutricional mostrou-se como um fator independentemente associado à sarcopenia, em que idosos abaixo do peso chegam a ser três vezes mais suscetíveis a doenças. ${ }^{14,27} \mathrm{~A}$ baixa ingesta de nutrientes, principalmente proteínas, pode ser a razão para o decréscimo da massa muscular e consequente limitação física identificada nesses indivíduos. $^{31}$

O dimorfismo sexual encontrado quando se observa o IMM foi notável, apresentando-se maior nas mulheres. Uma revisão sistemática identificou que muitos trabalhos descrevem a relação do decréscimo da MME com a faixa etária, etnia e sexo. ${ }^{32}$ Enquanto a massa muscular permanece regular nas mulheres até aproximadamente os 40 anos, os homens sofrem uma maior influência da idade, e perdem essa estabilidade entre os 20-30 anos. ${ }^{33,34}$

Assim, em estudos com o público extremamente idoso, é cada vez mais perceptível esse impacto do tempo na qualidade muscular no sexo masculino, podendo até mesmo ser ultrapassado pelo índice feminino. ${ }^{33,35}$

A avaliação da FV apresentou diferenças de acordo com o sexo, de modo que $55,1 \%$ dos homens e $77,8 \%$ das mulheres apresentaram pontuação inferior à nota de corte. Estudos anteriores divergem sobre qual grupo apresenta melhor desempenho, alguns indicando o grupo feminino, ${ }^{36,37}$ enquanto outros o masculino. ${ }^{38,39}$

Uma possível razão para o melhor desempenho dos homens pode ser um nível maior de escolaridade, o qual já se mostrou correlacionado ao índice, levando em consideração que todo contexto histórico educacional dos entrevistados coopera com essa divergência entre os gêneros em relação ao período escolar. ${ }^{39}$ Porém, como apresentado, a escolaridade foi semelhante entre os dois grupos.

O desempenho cognitivo foi observado por meio do teste de FV. Embora haja divergências e poucas evidências na literatura sobre a relação entre cognição e sarcopenia, ${ }^{40}$ estudos sugerem que a sarcopenia desempenhe um impacto negativo no desempenho cognitivo. ${ }^{41-44} \mathrm{Na}$ pesquisa em análise, no entanto, não se verificou correlação significativa e direta entre IMM e cognição.

É importante ressaltar que a sarcopenia pode estar associada a alguns domínios cognitivos específicos, de modo a não apresentar relação com a função cognitiva global. ${ }^{45}$

Resultados de um estudo coreano confirmam esse fato ao atestar que os domínios referentes à velocidade de processamento e à função executiva se relacionam à sarcopenia. ${ }^{46} \mathrm{~A}$ esfera cognitiva analisada pela pesquisa em discussão, a fluência verbal, foi positivamente relacionada à sarcopenia na literatura. ${ }^{45,47}$

Contudo, a amostra avaliada não se restringiu a idosos longevos, revelando a necessidade de ampliar as investigações 
sobre esse grupo. Ademais, uma metanálise assinalou que as associações entre sarcopenia e cognição variam segundo testes de função cognitiva e os instrumentos de medição da composição corporal usados. ${ }^{12}$ Outra ressalva se faz ao fato de que todos os idosos da presente amostra eram sarcopênicos e possuem baixo IMM, o que pode explicar a ausência da relação com a cognição.

Também não houve correlação significativa entre IMM e IMC, contrapondo dados presentes na literatura científica que associam baixo IMC com sarcopenia. ${ }^{48}$ Destaca-se, entretanto, que essa condição também pode ser visualizada em pessoas com obesidade, devido ao compartilhamento de mecanismos fisiopatológicos por ambas patologias. ${ }^{49}$

Acreditava-se, de fato, que a perda de massa muscular e de peso intrínseca ao envelhecimento ocasionava fraqueza em idosos. $^{5}$ Todavia, sabe-se que alterações na composição muscular, a exemplo de infiltrado de gordura no músculo, também são relevantes na qualidade e no desempenho muscular. ${ }^{5}$ Nesse contexto, os mecanismos envolvidos com a sarcopenia merecem ser melhor entendidos, a fim de que sua relação com o IMC seja esclarecida.

Em relação às medidas necessárias para atenuar o quadro sarcopênico, uma revisão destacou as vantagens do treinamento físico, somado à suplementação alimentar. ${ }^{50} \mathrm{~A}$ atividade física também desempenha impacto positivo nas funções cognitivas, por aprimorar a neuroplasticidade de determinadas estruturas cerebrais ao estimular mecanismos de angiogênese, sinaptogênese, neurogênese e liberação de neurotrofinas. ${ }^{51}$

É fundamental, diante disso, que as intervenções direcionadas à reabilitação sejam adaptadas para as particularidades vislumbradas na extrema longevidade, dentre as quais a independência no âmbito de autocuidado, a locomoção e a transferência. ${ }^{52}$ Sugerem-se exercícios que envolvam a sobrecarga gradual dos músculos, considerando-se as características fisiológicas do envelhecimento, com o intuito de evitar lesões. ${ }^{53}$

Dinâmicas de resistência são recomendadas, além de maior enfoque aos músculos dos membros inferiores, devido ao maior envolvimento na mobilidade, equilíbrio e marcha. ${ }^{53,54} \mathrm{O}$ desenvolvimento do sistema neuromuscular e a intensificação da taxa de síntese de proteínas constituem benefícios dessa modalidade de treinamento..$^{54}$

O estudo em discussão apresenta algumas limitações, como as diferenças entre as amostras das análises utilizadas para a comparação dos dados, uma vez que a literatura científica carece de estudos com idosos em extrema longevidade. Além disso, destaca-se a transversalidade da pesquisa, o que impossibilita inferir relações de causa e efeito entre os determinantes analisados. Soma-se ainda o viés de recrutamento, visto que muitos idosos não puderam ser entrevistados por problemas no endereço.

\section{CONCLUSÃO}

Os resultados apontaram alta prevalência de sarcopenia na amostra de idosos longevos. Não foi identificada relação entre IMM e CP, IMC e desempenho cognitivo.

Destaca-se correlação significativa entre IMC e CP. A ausência de correlação de IMM com CP e IMC sugere a necessidade de avaliar sarcopenia em idosos longevos a partir de métodos mais fidedignos.

A elevada prevalência da sarcopenia, acrescida às inúmeras lacunas na literatura, realçam a urgência de um olhar mais cuidadoso direcionado ao grupo de idosos longevos. Medidas de reabilitação, com destaque aos treinamentos de resistência, transferência, locomoção e autocuidado mostram-se fundamentais para amenizar esse quadro e conferir maior qualidade de vida a esse grupo etário, cada vez mais numeroso em decorrência da transição demográfica.

Cabe à comunidade científica intensificar os estudos com enfoque na longevidade extrema, bem como elucidar as variáveis relacionadas à sarcopenia e as diferenças de acordo com a faixa etária.

Esse conhecimento certamente favorece a compreensão da condição de saúde dos idosos longevos, cujo número é crescente diante da transição demográfica, de modo a prestar mais cuidado a eles e planejar intervenções preventivas, vinculando bem-estar à longevidade.

\section{AGRADECIMENTOS}

Aos alunos da Universidade Federal de Mato Grosso do Sul - UFMS pela colaboração na coleta de dados, e aos idosos participantes do estudo.

\section{REFERENCIAS}

1. Debia N, Silveira NDR. Indicadores socioculturais e histórias de vida de idosos longevos: heterogeneidade e ressignificações de hábitos na velhice. Rev Kairós Ger. 2019;22(1):291-305. Doi: https://doi.org/10.23925/2176901X.2019v22i1p291-305

2. Instituto Brasileiro de Geografia e Estatística. Estatísticas sociais [texto na Internet]. Rio de Janeiro: IBGE; c2017 [citado $2020 \mathrm{Abr}$ 19]. Disponível em: https://agenciadenoticias.ibge.gov.br/agencia-sala-deimprensa/2013-agencia-de-noticias/releases/18470-em2016-expectativa-de-vida-era-de-75-8-anos

3. Quaresma MLB, Ribeirinho C. Envelhecimento - desafios do Séc. XXI. Rev Kairós Ger. 2016;19(3):29-49. Doi: https://doi.org/10.23925/2176-901X.2016v19i3p29-49

4. Marzetti E, Calvani R, Tosato $M$, Cesari M, Di Bari M, Cherubini A, et al. Sarcopenia: an overview. Aging Clin Exp Res. 2017;29(1):11-17.

Doi:

http://dx.doi.org/10.1007/s40520-016-0704-5

5. Cruz-Jentoft AJ, Baeyens JP, Bauer JM, Boirie Y, Cederholm T, Landi F, et al. Sarcopenia: European consensus on definition and diagnosis: Report of the European Working Group on Sarcopenia in Older People. Age Ageing. 2010;39(4):412-23. Doi: http://dx.doi.org/10.1093/ageing/afq034

6. Chuang SY, Chang HY, Lee MS, Chia-Yu Chen R, Pan WH. Skeletal muscle mass and risk of death in an elderly population. Nutr Metab Cardiovasc Dis. 2014;24(7):78491. Doi: http://dx.doi.org/10.1016/j.numecd.2013.11.010

7. Srikanthan $P$, Karlamangla AS. Muscle mass index as a predictor of longevity in older adults. Am J Med. 2014;127(6):547-53.

http://dx.doi.org/10.1016/j.amjmed.2014.02.007 
8. Graf CE, Pichard C, Herrmann FR, Sieber CC, Zekry D, Genton L. Prevalence of low muscle mass according to body mass index in older adults. Nutrition. 2017;34:1249. Doi: http://dx.doi.org/10.1016/j.nut.2016.10.002

9. Landi F, Liperoti R, Russo A, Giovannini S, Tosato $M$, Capoluongo $E$, et al. Sarcopenia as a risk factor for falls in elderly individuals: results from the ilSIRENTE study. Clin Nutr. 2012;31(5):652-8.

Doi: http://dx.doi.org/10.1016/j.clnu.2012.02.007

10. Landi F, Liperoti R, Fusco D, Mastropaolo S, Quattrociocchi D, Proia A, et al. Sarcopenia and mortality among older nursing home residents. J Am Med Dir Assoc. 2012;13(2):121-6.

http://dx.doi.org/10.1016/i.jamda.2011.07.004

11. Peng TC, Chen $\mathrm{WL}, \mathrm{Wu}$ LW, Chang YW, Kao TW. Sarcopenia and cognitive impairment: A systematic review and meta-analysis. Clin Nutr. 2020;39(9):26952701. Doi: https://doi.org/10.1016/j.clnu.2019.12.014

12. Chang K, Hsu TH, Wu WT, Huang KC, Han DS. Association between sarcopenia and cognitive impairment: a systematic review and meta-analysis. J Am Med Dir Assoc. 2016;17(12):1164.e7-1164.e15.

Doi:

http://dx.doi.org/10.1016/j.jamda.2016.09.013

13. Aprahamian I, Cipolli GC, Yassuda MS. Sarcopenia and cognitive impairment: Possible physiopathological causation or just a spurious association?. Clin Nutr. 2020;39(5):1622.

http://dx.doi.org/10.1016/i.clnu.2020.03.027

14. Lee K, Shin Y, Huh J, Sung YS, Lee I. Recent issues on body composition imaging for sarcopenia evaluation. Korean Radiol. 2019;20(2):205-17. http://dx.doi.org/10.3348/kjr.2018.0479

15. Sergi G, Trevisan C, Veronese N, Lucato P, Manzato E. Imaging of sarcopenia. Eur J Radiol. 2016;85(8). Doi: http://dx.doi.org/10.1016/j.ejrad.2016.04.009

16. Rech CR, Dellagrana RA, Petroski MFN, Luiz ME. Validade de equações antropométricas para estimar a massa muscular em idosos. Rev Bras Cineantropom Desempenho Hum. 2012;14(1):23-31. Doi: http://dx.doi.org/10.5007/1980-0037.2012v14n1p23

17. Kawakami R, Murakami H, Sanada K, Tanaka N, Sawada SS, Tabata I, et al. Calf circumference as a surrogate marker of muscle mass for diagnosing sarcopenia in Japanese men and women. Geriatr Gerontol Int. 2014;15(8):969-76.

http://dx.doi.org/10.1111/ggi.12377

18. Peixoto LG, Barbosa CD, Nahas PC, Rossato LT, Oliveira EP. A circunferência da panturrilha está associada com a massa muscular de indivíduos hospitalizados. Rev Bras Nutr Clin. 2016;31(2):167-71.

19. Fukuoka $Y$, Narita T, Fujita $H$, Morii $T$, Sato $T$, Sassa MH, et al. Importance of physical evaluation using skeletal muscle mass index and body fat percentage to prevent sarcopenia in elderly Japanese diabetic patients. J Diabetes Investig. 2019;10(2):322-30. Doi: http://dx.doi.org/10.1111/jdi.12908

20. Guglielmi G, Ponti F, Agostini M, Amadori M, Battista G, Bazzocchi A. The role of DXA in sarcopenia. Aging Clin Exp Res. 2016;28(6):1047-60. Doi:
21. Instituto Brasileiro de Geografia e Estatística. Censo demográfico 2010. Características da população e dos domicílios: resultados do universo [texto na Internet]. Rio de Janeiro: IBGE; c2011 [citado 2020 Jan 20]. Disponível em:

https://biblioteca.ibge.gov.br/visualizacao/periodicos/93 Lcd 2010 caracteristicas populacao domicilios.pdf

22. Janssen I, Baumgartner RN, Ross $R$, Rosenberg $I H$, Roubenoff R. Skeletal muscle cutpoints associated with elevated physical disability risk in older men and women. Am J Epidemiol. 2004;15;159(4):413-21. Doi: http://dx.doi.org/10.1093/aje/kwh058

23. Brasil. Ministério da Saúde. Caderneta de saúde da pessoa idosa. 5 ed. Brasília (DF): Ministério da Saúde; 2018.

24. Brucki SM, Dozzi MSM, Fleury OIH, Bertolucci PHF. Dados normativos para o teste de fluência verbal categoria animais em nosso meio. Arq Neuro-Psiquiatr. 1997; 55(1):56-61. Doi: https://doi.org/10.1590/S0004282X1997000100009

25. Patel HP, Syddall HE, Jameson K, Robinson S, Denison $H$, Roberts $\mathrm{HC}$, et al. Prevalence of sarcopenia in community dwelling older people in the UK using the European Working Group on Sarcopenia in Older People (EWGSOP) definition: findings from the Hertfordshire Cohort Study (HCS). Age Ageing. 2013;42(3):378-84. Doi: http://dx.doi.org/10.1093/ageing/afs197

26. Viana LS, Macedo OG, Vilaça KHC, Garcia PA. Concordância de diferentes critérios de sarcopenia em idosas comunitárias. Fisioter Pesqui. 2018;25(2):151-7. Doi: 2950/17467625022018

27. Santos VR, Christofaro DGD, Gomes IC, Agostinete RR, Freitas Júnior IF, Gobbo LA. Factors associated with sarcopenia in subjects aged 80 years and over. Rev Nutr. 2015;28(3):319-26. Doi: https://doi.org/10.1590/1415$\underline{52732015000300008}$

28. Carmeli E. Frailty and primary sarcopenia: a review. Adv Exp Med Biol. 2017;1020:53-68. Doi: https://doi.org/10.1007/5584 201718

29. Pagotto V, Santos KF, Malaquias SG, Bachion MM, Silveira EA. Circunferência da panturrilha: validação clínica para avaliação de massa muscular em idosos. Rev Bras Enferm. 2018;71(2):322-8. Doi: https://doi.org/10.1590/00347167-2017-0121

30. Delmonico MJ, Harris TB, Visser M, Park SW, Conroy MB, Velasquez-mieyer $P$, et al. Longitudinal study of muscle strength, quality, and adipose tissue infiltration. Am J Clin Nutr. 2009;90(5):1579-85. Doi: https://doi.org/10.3945/ajcn.2009.28047

31. Barbosa-Silva MC. Subjective and objective nutritional assessment methods: What do they really assess? Curr Opin Clin Nutr Metab Care. 2008;11(3):248-54. Doi: https://doi.org/10.1097/mco.0b013e3282fba5d7

32. Schopf PP, Allendorf DB, Schwanke CHA, Gottlieb MGV. Idade, sexo, raça/etnia são fatores intrínsecos associados à perda de massa muscular: uma revisão sistemática. Rev Bras Cien Mov. 2017;25(2):195-204. Doi: http://dx.doi.org/10.18511/rbcm.v25i2.6333 
33. Silva AM, Shen W, Heo M, Gallagher D, Wang Z, Sardinha $B$, et al. Ethnicity-related skeletal muscle difference across the lifespan. Am J Hum Biol. 2010;22(1):76-82. Doi: https://doi.org/10.1002/ajhb.20956

34. Aleman-Mateo $\mathrm{H}$, Lee SY, Javed F, Thornton J, Heymsfield $\mathrm{SB}$, Pierson RN, et al. Elderly mexicans have less muscle and greater total and truncal fat compared to africanamericans and caucasians with the same BMI. J Nutr Heal Aging. 2009;13(10):919-23. Doi: https://doi.org/10.1007/s12603-009-0252-1

35. Woo J, Arai H, Ng TP, Sayer AA, Wong M, Syddall H, et al. Ethnic and geographic variations in muscle mass, muscle strength and physical performance measures. Eur Geriatr Med. 2014;5(3):155-64

Doi:

https://doi.org/10.1016/i.eurger.2014.04.003

36. Lezak MD, Howieson DB, Loring DW, Hannay HJ, Fischer JS. Neuropsychological assessment. $4^{\text {th }}$ ed. New York: Oxford University Press; 2004.

37. Crossley M, D'Arcy C, Rawson NS. Letter and category fluency in community-dwelling Canadian seniors: A comparison of normal participants to those with dementia of the Alzheimer or vascular type. J Clin Exp Neuropsychol. 1997;19(1):52-62. Doi: https://doi.org/10.1080/01688639708403836

38. Kempler D, Teng EL, Dick M, Taussig IM, Davis DS. The effects of age, education, and ethnicity on verbal fluency. J Int Neuropsychol Soc. 1998;4(6):531-8. Doi: https://doi.org/10.1017/s1355617798466013

39. Silva TBL, Yassuda MS, Guimarães VV, Florindo AA. Fluência verbal e variáveis sociodemográficas no processo de envelhecimento: um estudo epidemiológico. Psicol Reflex Crit. 2011;24(4):739-46. Doi: https://doi.org/10.1590/S0102-79722011000400014

40. Cipolli GC. Sarcopenia e comprometimento cognitivo em idosos longevos: resultados do estudo FIBRA [Dissertação]. São Paulo: Universidade de São Paulo, Escola de Artes, Ciências e Humanidades; 2019. Doi: https://doi.org/10.11606/D.100.2019.tde-05082019220140

41. Nishiguchi S, Yamada M, Shirooka H, Nozaki Y, Fukutani N, Tashiro $Y$, et al. Sarcopenia as a risk factor for cognitive deterioration in community-dwelling older adults: a 1year prospective study. J Am Med Dir Assoc. 2016;16(2):120-4.

Doi: https://doi.org/10.1016/i.jamda.2015.12.096

42. Gao L, Jiaojiao J, Yang M, Hao Q, Luo L, Dong B. Prevalence of sarcopenia and associated factors in chinese community-dwelling elderly: comparison between rural and urban areas. J Am Med Dir Assoc. 2015;16(11):1003.e1-1003.e6.

Doi: https://doi.org/10.1016/i.jamda.2015.07.020

43. Alexandre TS, Duarte YA, Santos JL, Wong R, Lebrão ML. Prevalence and associated factors of sarcopenia among elderly in Brazil: findings from the SABE study. J Nutr Health Aging. 2014;18(3):284-90. Doi: https://doi.org/10.1007/s12603-013-0413-0
44. Ogawa Y, Kaneko Y, Sato T, Shimizu S, Kanetaka H, Hanyu $\mathrm{H}$. Sarcopenia and muscle functions at various stages of Alzheimer disease. Front Neurol. 2018;9:710. Doi: https://doi.org/10.3389/fneur.2018.00710

45. Huang $C$, Hwang $A$, Liu L, Chen L, Peng L, Chen L, et al. Association of dynapenia, sarcopenia and cognitive impairment among community-dwelling older Taiwanese. Rejuvenation Res. 2015;19(1):71-8. Doi: https://doi.org/10.1089/rej.2015.1710

46. Kim M, Won CW. Sarcopenia is associated with cognitive impairment mainly due to slow gait speed: results from the Korean Frailty and Aging Cohort Study (KFACS). Int J Env Res Public Heal. 2019;16(9):1491. Doi: https://doi.org/10.3390/ijerph16091491

47. Szlejf C, Suemoto CK, Lotufo PA, Benseñor IM. Association of sarcopenia with performance on multiple cognitive domains: results from the ELSA-Brasil Study. J Gerontol A Biol Sci Med Sci. 2019 4;74(11):1805-1811. Doi: https://doi.org/10.1093/gerona/glz118

48. Marty E, Liu Y, Samuel A, Or O, Lane J. A review of sarcopenia: Enhancing awareness of an increasingly prevalent disease. Bone. 2017;105:276-86. Doi: https://doi.org/10.1016/i.bone.2017.09.008

49. Choi KM. Sarcopenia and sarcopenic obesity. Korean J Intern Med. 2016;31:1054-60. Doi: https://doi.org/10.3904/kjim.2016.193

50. Denison HJ, Cooper C, Sayer AA, Robinson SM. Prevention and optimal management of sarcopenia: a review of combined exercise and nutrition interventions to improve muscle outcomes in older people. Clin Interv Aging. 2015;10:859-69.

Doi:

https://doi.org/10.2147/CIA.S55842. eCollection 2015

51. Hötting K, Röder B. Beneficial effects of physical exercise on neuroplasticity and cognition. Neurosci Biobehav Rev. 2013;37(9 Pt $\quad$ B):2243-57. Doi: https://doi.org/10.1016/i.neubiorev.2013.04.005

52. Fernandes DS, Gonçalves LHT, Ferreira AMR, Santos MIP de O. Avaliação da capacidade funcional de idosos longevos amazônidas. Rev Bras Enferm. 2019;72(Suppl 2):55-61. Doi: https://doi.org/10.1590/0034-7167-2017$\underline{0798}$

53. Giallauria F, Cittadini A, Smart NA, Vigorito C. Resistance training and sarcopenia. Monaldi Arch Chest Dis. 2016;84(1-2):738. Doi: https://doi.org/10.4081/monaldi.2015.738

54. Dhillon RJ, Hasni S. Pathogenesis and Management of Sarcopenia. Clin Geriatr Med. 2017;33(1):17-26. Doi: https://doi.org/10.1016/i.cger.2016.08.002 